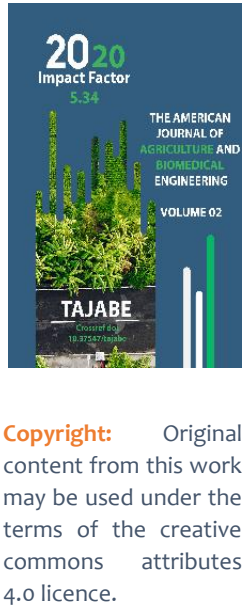

\title{
The Growth And Development Of Crossed Varieties F2 Obtained From Medium Fiber Varieties Of Cotton
}

\author{
Namazov Shadman Ergashevich \\ Doctor Of Agricultural Sciences, Professor Of Scientific Research Institute Of Breeding, Seed \\ Production And Agricultural Technology Of Cotton. Tashkent, Uzbekistan \\ Abdumalikov Ulugbek Ziyodilla ugli \\ Researcher Of Scientific Research Institute Of Breeding, Seed Production And Agricultural \\ Technology Of Cotton, Tashkent, Uzbekistan
}

\section{ABSTRACT}

This article examines the growth and development of crossed varieties of F2 cotton. Hybrids of medium-fiber cotton varieties were studied at field experimental sites of the research and experimental center located in the Kuva district of the Fergana region.

\section{KEYWORDS}

Cotton, fruit branches, variant, varieties, donor.

\section{INTRODUCTION}

Today, the process of modernization and development of agriculture is increasing in the Republic of Uzbekistan. Increasing scientific potential and innovative achievements contribute to the development of human civilization. In particular, the creation of new crossed cotton varieties, their in-depth study and analysis will serve as a driving force for the development of agriculture.

To create cotton varieties that meet modern requirements, a variety of well-studied primary sources (donors) are needed. The varieties created in recent years and introduced into practice are characterized by positive morpho-biological characteristics and technological quality indicators. It is important to use new primary sources (donors) when creating such varieties. ...

\section{MATERIALS AND METHODS}

According to the experiments, the memory of H. Egamov, I. Kimsanov, S. Rasulov, N. Mirkhamidova, U. Abdumalikov (2015-2017), with a continuous selection process, a new cotton variety has an advantage over the previous ones by $10-15 \%$ and there is a need to 
use this is in practice. [1, Uzb. agricultural Journal 2018, No. 5, 32 p.].

According to the experiments of $U$. Abdumalikov, Sh. Namazov, S. Matekubov (2019), the germination energy and fertility of cotton seeds have a direct influence on the subsequent stages of plant development. [2, Agro ilm, 2020, No. 3, 3p.].

Our research (2020) was carried out in the field experimental plots of the Research Institute of Breeding, Seed Production and Agricultural Technology of Cotton Growing, located in the Kuva district of the Fergana region. In the experimental field, the growth and development of new crossed F2 varieties obtained from medium-fiber cotton varieties were studied. When we examined our hybrids on the basis of data obtained from the observation on June 1 (table 1), we found that the highest growing cultivars were found $\mathrm{F}_{2}$ (Andes-36 x Andes-37) $19.1 \mathrm{~cm}, \mathrm{~F} 2$ (Sulton $\mathrm{x}$ Andes -36) $18.6 \mathrm{~cm}, \mathrm{~F} 2$ (H. JarkurganAnd-37) $18.6 \mathrm{~cm}, \mathrm{~F} 2$ ( $\mathrm{H}$. Jarkurgan And-36) $18.8 \mathrm{~cm}$, Relatively low F2 (And-36 x Omad) $14.5 \mathrm{~cm}, \mathrm{~F} 2$
(Andes-36 x Turon) $15.4 \mathrm{~cm}$, F2 (Jarkurgan x Omad) $15.3 \mathrm{~cm}, \mathrm{~F} 2$ (Bukh-102 x Omad) 14.3 $\mathrm{cm}$. When we checked the number of leaves, the crossed variety F2 (Bukh-102 x Omad) produced the smallest number of leaves, the number of which was 3.1 leaves, the number of leaves of high-grade crossed varieties averaged 4.4 leaves, and the number of leaves of the most productive variety (hybrid) F2 (Andes-36 x Andes-37) was 4.7 leaves per plant.

\section{RESULTS AND DISCUSSIONS}

According to the July observations of our crossed varieties (Andes-36 x Omad), F2 (Ande-36 x Turon), F2 (Jarkurgan $x$ Omad), F2 (Bukh-102 x Omad) (table-1), the height of the plants of the above crossed varieties are 5-10 $\mathrm{cm}$ lower compared to other crossed varieties (hybrids). In the 1st, 12th, 18th, 19th, 21st, 22nd, 23rd, 28th, In the 32nd, 34th, 41st, 44th variants, more than 7 fruit branches were found. In the remaining variants, fruit branches are 1-2 less than in other variants.

Growth and development of crossed F2 cotton varieties during the growing season

\section{Table 1}




\begin{tabular}{|c|c|c|c|c|c|c|c|}
\hline \multirow[b]{2}{*}{ № } & \multirow[b]{2}{*}{ Names of crossed variants } & \multirow{2}{*}{$\begin{array}{l}\text { date } \\
100 \% \\
\text { seed } \\
\text { germ } \\
\text { inati } \\
\text { on }\end{array}$} & \multicolumn{2}{|l|}{1.06} & \multicolumn{3}{|l|}{1.07} \\
\hline & & & $\begin{array}{l}\text { Height } \\
\text { of } \\
\text { growt } \\
\mathrm{h} \\
\mathrm{cm}\end{array}$ & $\begin{array}{l}\text { Numb } \\
\text { er of } \\
\text { leaves } \\
\text { pieces }\end{array}$ & $\begin{array}{l}\text { Heig } \\
\text { ht of } \\
\text { grow } \\
\text { th, } \\
\mathrm{cm}\end{array}$ & $\begin{array}{l}\text { Fruit } \\
\text { bran } \\
\text { ches, } \\
\text { piece } \\
\text { s }\end{array}$ & $\begin{array}{l}\text { Fruit } \\
\text { eleme } \\
\text { nts, } \\
\text { pieces }\end{array}$ \\
\hline 1 & F2 (Andij-36x Andij -37) & $\begin{array}{l}22.0 \\
4\end{array}$ & 19,1 & 4,7 & 48,7 & 7,5 & 7,8 \\
\hline 2 & F2 (Andij-36x S-6524) & $\begin{array}{l}24.0 \\
4\end{array}$ & 18,2 & 4,3 & 47,4 & 6,8 & 7,6 \\
\hline 3 & F2 ( Andij -36x Nam-34) & $\begin{array}{l}22.0 \\
4\end{array}$ & 17,6 & 4,1 & 47,2 & 6,3 & 6,8 \\
\hline 4 & F2 (Andij -36xNam-77) & $\begin{array}{l}24.0 \\
4\end{array}$ & 17,3 & 3,9 & 46,6 & 5,9 & 6,2 \\
\hline 5 & F2 (Andij-36x Omad) & $\begin{array}{l}22.0 \\
4\end{array}$ & 14,5 & 3,2 & 34,6 & 5,4 & 5,8 \\
\hline 6 & F2 (Andij-36x Sulton) & $\begin{array}{l}24.0 \\
4\end{array}$ & 17,8 & 4,2 & 48,2 & 6,8 & 7,4 \\
\hline & & 24.0 & & & & & \\
\hline$s^{7} \mathrm{~J} J \mathrm{O}$ & 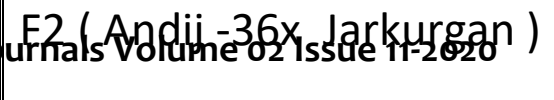 & 4 & 17,2 & 4,5 & 47,8 & 6,7 & 6,4 \\
\hline
\end{tabular}


The American Journal of Agriculture and Boimedical Engineering (ISSN - 2689-1018)

Published: November 30, 2020 | Pages: 74-81

\begin{tabular}{|c|c|c|c|c|c|c|c|}
\hline 8 & F2 (Andij -36x Kelajak) & $\begin{array}{l}22.0 \\
4\end{array}$ & 15,8 & 3,4 & 45,7 & 5,7 & 6,2 \\
\hline 9 & F2 (Andij-36xBukh-102) & $\begin{array}{l}24.0 \\
4\end{array}$ & 16,2 & 3,8 & 46,4 & 6,2 & $\mid 7,3$ \\
\hline 10 & F2 (Andij-36xTuron) & $\begin{array}{l}24.0 \\
4\end{array}$ & 15,4 & 3,8 & 45,8 & 6,5 & $\mid 6,8$ \\
\hline 11 & F2 (Andij-36xUzPITI-201) & $\begin{array}{l}22.0 \\
4\end{array}$ & 16,3 & 3,2 & 46,3 & 6,3 & 7,4 \\
\hline 12 & F2 (Sulton xAndij-37) & $\begin{array}{l}22.0 \\
4\end{array}$ & 17,7 & 4,4 & 47,5 & 7,4 & $\mid 7,7$ \\
\hline 13 & F2 (Sulton xS-6524) & $\begin{array}{l}24.0 \\
4\end{array}$ & 16,9 & 3,6 & 43,2 & 5,8 & 6,7 \\
\hline 14 & F2 (Sulton xNam-34) & $\begin{array}{l}22.0 \\
4\end{array}$ & 16,7 & 3,4 & 44,9 & 6,0 & | 7,6 \\
\hline 15 & F2 (Sulton x Nam-77) & $\begin{array}{l}24.0 \\
4\end{array}$ & 17,1 & 4,1 & 45,2 & 6,2 & $\mid 6,9$ \\
\hline 16 & F2 (Sulton xOmad) & $\begin{array}{l}22.0 \\
4\end{array}$ & 16,8 & 3,8 & 42,3 & 6,2 & 6,6 \\
\hline 17 & F2 (Sulton xTuron) & $\begin{array}{l}24.0 \\
4\end{array}$ & 17,3 & 3,5 & 43,6 & 6,1 & 6,2 \\
\hline
\end{tabular}


The American Journal of Agriculture and Boimedical Engineering (ISSN - 2689-1018)

Published: November 30, 2020 | Pages: 74-81

\begin{tabular}{|c|c|c|c|c|c|c|c|}
\hline 18 & F2 (Sulton x Jarkurgan ) & $\begin{array}{l}22.0 \\
4\end{array}$ & 18,7 & 4,6 & 47,4 & 7,6 & 7,5 \\
\hline 19 & F2 (Sulton xUzPITI-201) & $\begin{array}{l}24.0 \\
4\end{array}$ & 17,2 & 4,2 & 47,2 & 7,3 & $\mid 7,3$ \\
\hline 20 & F2 (Sulton xKelajak) & $\begin{array}{l}22.0 \\
4\end{array}$ & 16,7 & 3,7 & 45,4 & 6,7 & $\mid 6,8$ \\
\hline 21 & F2 (Sulton xBukh-102) & $\begin{array}{l}22.0 \\
4\end{array}$ & 17,6 & 3,8 & 47,8 & 7,4 & 7,1 \\
\hline 22 & F2 (Sulton xAndij-36) & $\begin{array}{l}24.0 \\
4\end{array}$ & 18,6 & 4,5 & 48,3 & 7,8 & $\mid 7,4$ \\
\hline 23 & F2 (Jarkurgan x And-37) & $\begin{array}{l}22.0 \\
4\end{array}$ & 18,6 & 4,3 & 47,8 & 7,4 & 7,6 \\
\hline 24 & F2 ( Jarkurgan x S-6524) & $\begin{array}{l}24.0 \\
4\end{array}$ & 17,7 & 3,8 & 47,4 & 6,2 & 7,2 \\
\hline 25 & F2 ( Jarkurgan x Nam-34) & $\begin{array}{l}22.0 \\
4\end{array}$ & 18,1 & 3,9 & 48,2 & 6,5 & $\mid 6,9$ \\
\hline 26 & F2 (Jarkurgan x Omad) & $\begin{array}{l}24.0 \\
4\end{array}$ & 15,3 & 3,2 & 38,6 & 6,1 & 6,4 \\
\hline 27 & F2 (Jarkurgan x Bukh-102) & $\begin{array}{l}22.0 \\
4\end{array}$ & 18,5 & 4,2 & 46,9 & 6,4 & 5,7 \\
\hline
\end{tabular}


The American Journal of Agriculture and Boimedical Engineering (ISSN - 2689-1018)

Published: November 30, 2020 | Pages: 74-81

\begin{tabular}{|c|c|c|c|c|c|c|c|}
\hline 28 & F2 (Jarkurgan x Sultan) & $\begin{array}{l}22.0 \\
4\end{array}$ & 18,3 & 4,1 & 48,2 & 7,3 & 7,8 \\
\hline 29 & F2 (Jarkurgan x Kelajak) & $\begin{array}{l}24.0 \\
4\end{array}$ & 16,3 & 3,5 & 45,8 & 6,7 & $\mid 6,4$ \\
\hline 30 & F2 ( Jarkurgan x Nam-77) & $\begin{array}{l}24.0 \\
4\end{array}$ & 17,6 & 3,4 & 46,8 & 6,2 & $\mid 6,8$ \\
\hline 31 & F2 (Jarkurgan x Turon) & $\begin{array}{l}24.0 \\
4\end{array}$ & 16,5 & 3,6 & 46,5 & 5,9 & 6,3 \\
\hline 32 & F2 ( Jarkurgan x Andiz-36) & $\begin{array}{l}24.0 \\
4\end{array}$ & 18,8 & 4,2 & 48,3 & 7,5 & $\mid 7,8$ \\
\hline 33 & $\begin{array}{l}\text { F2 ( Jarkurgan x UzPITI- } \\
\text { 201) }\end{array}$ & $\begin{array}{l}24.0 \\
4\end{array}$ & 17,2 & 3,8 & 46,8 & 6,4 & 6,6 \\
\hline 34 & F2 (Bukh-102xAndij-37) & $\begin{array}{l}22.0 \\
4\end{array}$ & 17,8 & 3,4 & 47,5 & 7,4 & $\mid 7,3$ \\
\hline 35 & F2 (Bukh-102xS-6524) & $\begin{array}{l}22.0 \\
4\end{array}$ & 16,9 & 3,5 & 43,4 & 6,4 & $\mid 7,4$ \\
\hline 36 & F2 (Bukh-102xNam-34) & $\begin{array}{l}22.0 \\
4\end{array}$ & 16,8 & 3,4 & 44,9 & 6,2 & 7,1 \\
\hline 37 & F2 (Bukh-102xOmad) & $\begin{array}{l}22.0 \\
4\end{array}$ & 14,3 & 3,1 & 37,7 & 6,0 & 6,9 \\
\hline
\end{tabular}


The American Journal of Agriculture and Boimedical Engineering (ISSN - 2689-1018)

Published: November 30, 2020 | Pages: 74-81

\begin{tabular}{|c|c|c|c|c|c|c|c|}
\hline 38 & F2 (Bukh-102xUzPITI-201) & $\begin{array}{l}22.0 \\
4\end{array}$ & 16,8 & 3,4 & 43,3 & 6,5 & 6,4 \\
\hline 39 & F2 (Bukh-102xSulton) & $\begin{array}{l}22.0 \\
4\end{array}$ & 17,8 & 3,8 & 47,2 & 7,0 & 7,3 \\
\hline 40 & F2 (Bukh-102xKelajak) & $\begin{array}{l}24.0 \\
4\end{array}$ & 17,2 & 3,6 & 44,4 & 6,6 & 6,4 \\
\hline 41 & F2 (Bukh-102xNam-77) & $\begin{array}{l}22.0 \\
4\end{array}$ & 17,1 & 3,5 & 47,2 & 7,3 & 6,7 \\
\hline 42 & F2 (Bukh-102x Jarkurgan ) & $\begin{array}{l}24.0 \\
4\end{array}$ & 16,8 & 3,2 & 46,9 & 6,9 & 7,2 \\
\hline 43 & F2 (Bukh-102xTuron) & $\begin{array}{l}22.0 \\
4\end{array}$ & 16,6 & 3,2 & 46,8 & 6,8 & 6,6 \\
\hline 44 & F2 (Bukh-102xAndij-36) & $\begin{array}{l}24.0 \\
4\end{array}$ & 18,4 & 4,2 & 47,7 & 7,5 & 7,0 \\
\hline
\end{tabular}

When calculating the yield, it was found that in options $1,2,12,14,28$ by 7.5 pieces more than in other crossed varieties (hybrids), and in 5, 27 options by 5.8, and 5.7 pieces - less than the above varieties.

\section{CONCLUSION}

Thus, when we analyzed the height of plants, the number of leaves, branches and fruit elements of the crossed varieties, we found that the highest growing of the studied crossed varieties were F2 (Andes-36 x Andes37) $19.1 \mathrm{~cm}, \mathrm{~F} 2$ (Sulton $x$ Andes-36) 18.6. cm, F2
(Jarkurgan x And-37 7) 18.6 cm, F2 (Jarkurgan x A-36) $18.8 \mathrm{~cm}$. The largest number of leaves was formed by crossed variants F2 (Andes-36 $x$ Andes-37), which are relatively other varieties produced 4.7 more leaves. When calculating the yield, it was found that in options 1, 2, 12, 14, 28, 7.5 pieces more than in other crossed options (hybrids). 
The American Journal of Agriculture and Boimedical Engineering (ISSN - 2689-1018)

Published: November 30, 2020 | Pages: 74-81

Doi: https://doi.org/10.37547/tajabe/Volumeo2Issue11-14

\section{REFERENCES}

1. X.Egamov, I.Kimsanov, S.Rasulov, N.Mirkhamidova, U.Abdumalikov. Identification of useful economic features of new cotton fields // Agriculture of Uzbekistan, 2018, №5, p.32

2. U.Abdumalikov, Sh.Namazov, S.Matyoqubov. Determination of fertility of $\mathrm{F} 1$ hybrids from medium-fiber cotton varieties // Agro ilm, 2020, №3, p.3 